\title{
APLICAÇÃO DO PROJETO DE EXPERIMENTOS PARA OTIMIZAÇÃO DE UMA INOVAÇÃO TECNOLÓGICA
}

\section{APPLICATION OF A DESIGN OF EXPERIMENTS FOR OPTIMIZATION OF A TECHNOLOGICAL INNOVATION}

\author{
Mariela Haidée Aranda ${ }^{1}$; Carlos Fernando $\mathrm{Jung}^{2}$; Carla Schwengber ten Caten ${ }^{3}$ \\ ${ }^{1}$ Universidade Federal do Rio Grande do Sul - UFRGS - Porto Alegre - Brasil \\ mariela@producao.ufrgs.br \\ ${ }^{2}$ Universidade Federal do Rio Grande do Sul - UFRGS - Porto Alegre - Brasil jung@,faccat.br \\ ${ }^{3}$ Universidade Federal do Rio Grande do Sul - UFRGS - Porto Alegre - Brasil \\ tencaten@producao.ufrgs.br
}

\begin{abstract}
Resumo
Este artigo apresenta os resultados da aplicação da técnica de planejamento de experimentos (DOE) em uma inovação de média complexidade tecnológica. Trata-se de uma churrasqueira portátil, construída a partir da reutilização de um botijão de gás para refrigeração considerado como resíduo após a utilização do gás. O objetivo do experimento foi determinar quais os fatores que influenciam na variável de resposta tempo de cocção para assar distintos tipos de carne na churrasqueira portátil. O planejamento do experimento foi realizado utilizando-se a técnica de Projeto de Experimentos. Foi utilizado um projeto fatorial fracionado $2^{5-1}$ sem repetições contemplando 16 ensaios. Os resultados foram analisados utilizando-se a análise de regressão onde foram investigadas, além dos fatores principais, as interações de dois fatores. Com a equação de regressão é possível prever os tempos de cocção para cada uma das combinações dos níveis dos fatores controláveis.
\end{abstract}

Palavras-chave: projeto de experimento; inovação, regressão linear, Projetos $2^{\mathrm{k}-\mathrm{p}}$, otimização.

\section{Introdução}

A flexibilidade e a adaptabilidade são atributos cada vez mais essenciais à prática da engenharia. Com as freqüentes mudanças de tecnologias e a constante demanda por produtos inovadores, os engenheiros devem estar aptos a utilizar e adaptar qualquer ferramenta para a otimização de produtos sejam eles de baixa, média ou alta complexidade tecnológica.

Para Colenci (2000) esta situação reflete a atual necessidade do engenheiro torna-se flexível para aprender a aprender maneiras de desenvolver, adequar e aplicar teorias e técnicas a novos ambientes e problemas do dia-a-dia. Assim, o profissional de engenharia do século XXI deve ter habilidades para resolver problemas em qualquer situação ou grau de complexidade. 
Pizzolato, Caten e Flogliatto (2005) afirmam que para manter seus produtos no mercado com os níveis demandados de qualidade, empresas industriais e engenheiros de produtos necessitam de métodos, metodologias e ferramentas eficazes para o desenvolvimento e otimização de produtos.

A utilização do planejamento de experimentos pode contribuir com o desenvolvimento industrial pela otimização das grandezas de interesse, pela determinação dos fatores influentes sobre essas grandezas e, eventualmente, pelas suas interações e minimização dos efeitos da variabilidade sobre o desempenho de um processo ou produto (BONDUELLE, 1994).

Este artigo apresenta os resultados da aplicação da técnica de planejamento e análise de experimentos a uma inovação de média complexidade tecnológica. Trata-se de uma churrasqueira portátil, construída a partir da reutilização de um botijão de gás para refrigeração que é considerado um resíduo após a utilização do gás. Os objetivos do trabalho foram: (i) a utilização do projeto de experimentos para analisar os fatores que possuem influência na variável de resposta tempo de cocção para assar distintos tipos de carne nessa churrasqueira portátil, e (ii) demonstrar a possibilidade da aplicação desta técnica em uma inovação incremental de média complexidade tecnológica.

\section{Referencial teórico}

\subsection{Inovação tecnológica e produtos de baixa, média e alta complexidade tecnológica}

Há diferentes concepções sobre o que é uma inovação, afirma Hord (1987) que uma inovação é qualquer aspecto novo para um indivíduo dentro de um sistema. Para Hernández et al. (2000), uma inovação pode não ser considerada como tal por determinada pessoa e, por outra, ser vista como uma coisa nova, dentro do mesmo sistema. Por esta razão, o problema na hora de aproximar-se da temática da inovação não é tanto a definição que importa, mas a interpretação, ou seja, o reconhecimento do ponto de vista do qual parte a inovação e, a quem esta se destina. Esse autor refere ainda que a inovação não é a mesma coisa para quem a desenvolve, para quem a distribui e para quem a recebe. Portanto, a definição do que constitui uma inovação resulta da confluência de uma pluralidade de opiniões que procedem dos que têm algum tipo de relação com a inovação.

A inovação segundo Hennig (1994) significa: enxergar o novo no velho; é criar novos modelos. Nesse contexto, a inovação de produto ou processo significa a introdução de novas tecnologias e melhorias ou mudanças em produtos e processos existentes. As inovações de produto ou processo envolvem uma série de atividades científicas, tecnológicas, organizacionais, financeiras e comerciais. 
As inovações em produtos podem ser classificadas a partir de vários critérios, sendo que o mais usual é classificar em função do grau de mudança que o novo produto representa em relação aos existentes. Rozenfeld et al. (2006) afirmam que os projetos que representam inovações podem ser classificados em: (i) radicais, que envolvem significativas modificações no produto existente, necessitando a utilização de novas tecnologias e materiais, podendo gerar uma nova categoria ou família de produtos para a empresa; (ii) de plataforma ou próxima geração, que apresentam importantes alterações, mas, que não necessitam a utilização de novas tecnologias, sendo que estão associados a obtenção de novas soluções para o cliente e representam a próxima geração de produtos da empresa; e (iii) incrementais ou derivados, que surgem a partir de pequenas modificações em relação aos já existentes no portfólio de produtos da empresa ou mercado, normalmente originam-se na necessidade de redução de custo, ou na oferta de novas facilidades funcionais e operacionais ao usuário.

Bertoncello e Gomes (2002) afirmam que um novo produto de baixa complexidade tecnológica permite que quase todos os problemas sejam resolvidos individualmente pelo projetista. Isto ocorre quando o novo produto é resultante do projeto cuja solução funcional e formal não está contida no atual estado da arte, ou seja, não existe produto similar industrializado ou comercializado. Em produtos de média complexidade tecnológica, já existe a necessidade do projetista buscar auxílio de profissionais de outras áreas, para a resolução de problemas específicos, estes não incluídos em sua esfera de competência. Nos produtos de alta complexidade tecnológica, o projetista apenas participa da resolução de alguns componentes do produto e de forma mais ampla na abordagem conceitual e metodológica.

\subsection{A inovação objeto do experimento}

Necessidades que possam gerar um novo produto podem ser identificadas no próprio contexto diário de trabalho, em ambientes de lazer, supermercados, livrarias e lojas em geral, como também, problemas gerados por descarte de produtos após sua utilização, considerados como resíduos, representam excelentes oportunidades à inovação. Bonsiepe (1978), afirma que a observação de atividades, como fundamento para o descobrimento de uma necessidade, significa o momento em que se registra uma situação em forma de uma necessidade insatisfeita num grupo ou numa coletividade.

Corroborando, Kuczmarski (1998) afirma que inicialmente devem ser identificadas necessidades e problemas no mercado, para, posteriormente serem transformadas em produtos que representem soluções. Para Danilevics (2006) a esta estratégia estaria associada a lógica da inovação. 
A inovação, objeto desse estudo experimental, foi desenvolvida por um aluno do Curso de Engenharia de Produção da Faccat - Faculdades Integradas de Taquara, RS, que identificou um problema de ordem ambiental e, ao mesmo tempo, visualizou uma nova oportunidade de negócio. $\mathrm{O}$ aluno percebeu que os recipientes (botijões) que acondicionam gás, destinados a equipamentos de refrigeração, eram descartados em depósitos de resíduos ocasionando um sério problema ambiental.

A partir disso, o aluno teve a idéia de aproveitar o resíduo para ser desenvolvido um novo produto, uma inovadora churrasqueira portátil, como pode ser visto na Figura 1. Esse produto passou a ser desenvolvido e fabricado pelo próprio aluno que além de aproveitar os resíduos existentes em grande quantidade na região, aumentou a sua renda mensal pela comercialização desse produto.

Figura 1 - (i) botijão (resíduo) e (ii) churrasqueira portátil (produto obtido)

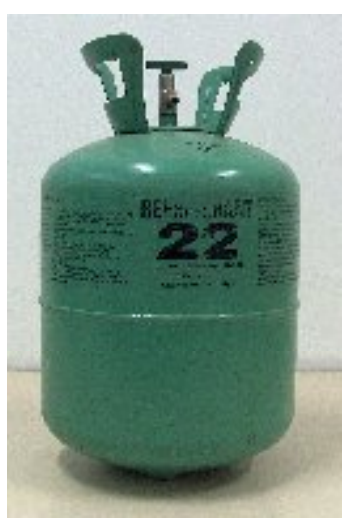

(i)

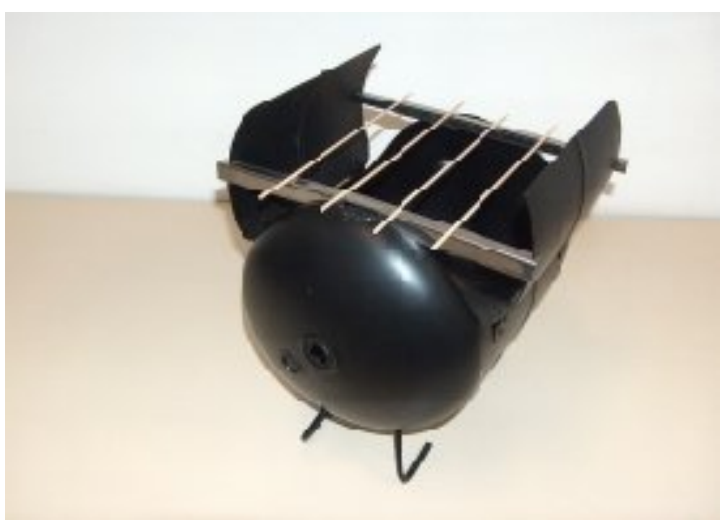

(ii)

\subsection{Projeto de experimentos}

Galdámez (2002) afirma que a Estatística, como ciência, possui inúmeras aplicações para a melhoria de sistemas produtivos. Nas empresas a Estatística pode ser utilizada como ferramenta para controle e melhoria da qualidade. Exemplos destas feramentas são: (i) Seis Sigma, (ii) Projeto de Experimentos - Design of Experiments, (iii) Análise de Variância - ANOVA, (iv) Controle Estatístico do Processo - CEP, (v) Metodologia de Superfície de Resposta - MSR, e (vi) Reprodutividade e Repetibilidade - R\&R.

Para que um experimento seja realizado de forma eficiente e produza resultados eficazes, deve-se empregar métodos científicos no seu planejamento (MONTGOMERY, 1997). Nesse contexto, o Projeto de Experimentos (DOE) é uma das ferramentas para se obter uma melhor performance das características da qualidade, tendo por resultado uma maior capacidade competitiva do produto no mercado (ELSAYED; CHEN, 1993; PHADKED, 1989).

O Planejamento de Experimentos (Design of Experiments, DOE) é uma técnica utilizada para se planejar experimentos, ou seja, para definir quais dados, em que quantidade e em que 
condições devem ser coletadas durante um determinado experimento, buscando, basicamente, satisfazer dois grandes objetivos: a precisão estatística possível na resposta e o menor custo. Atualmente, essa técnica vem sendo utilizada em grande escala.

Corroborando, Ribeiro e Caten (2003) afirmam que através dela, pesquisadores podem determinar os fatores do processo que exercem maior influência no desempenho de um determinado processo, tendo como resultados: (i) a redução da variação do processo e aumento da concordância entre os valores nominais obtidos e os valores pretendidos; (ii) a redução do tempo do processo; (iii) a redução do custo operacional; e (iv) a melhoria no rendimento do processo.

O experimento planejado é um teste ou uma série de testes nos quais se induzem mudanças deliberadas ou estímulos nas variáveis de entrada (inputs) em um processo ou sistema, de forma que seja possível observar e identificar os efeitos nas variáveis de saída (outputs), veja a Figura 2. O processo ou sistema de transformação é representado pela combinação de máquinas, métodos, pessoas e outros recursos que transformam determinadas varáveis de entrada em resultados que representam melhorias em produtos ou processos (GALDÁMEZ, 2002).

\section{Figura 2 - Modelo básico de um processo ou sistema}

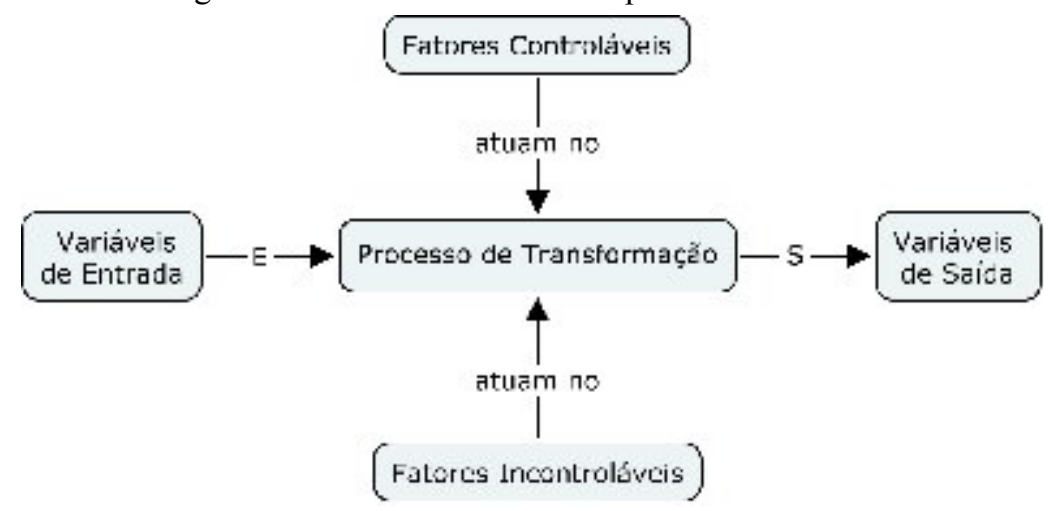

Fonte: Adaptado de Galdámez (2002)

Os três princípios básicos do planejamento de experimentos são representados por replicação, aleatoriedade e blocagem. Fazer um experimento com réplicas é importante por dois motivos: (i) permite a obtenção do erro experimental, a estimativa desse erro é básica para verificar se as diferenças observadas nos dados são estatisticamente significativas; e (ii) pelo fato de que se a média de uma amostra for utilizada para estimar o efeito de um fator no experimento, a replicação permite a obtenção de uma estimativa mais precisa desse fator (MONTGOMERY, 1997). Os experimentos, com suas réplicas devem ser realizados de forma aleatória, de modo a garantir a mesma distribuição de todos os fatores não controlados no experimento.

Quando o número de fatores a serem investigados aumenta, o número de ensaios a serem realizados no experimento também aumenta, exigindo um maior investimento e tempo para a sua execução. Nestes casos se utilizam técnicas como blocagem e/ou fracionamento. A blocagem 
permite que o experimento seja realizado em diferentes dias ou em diferentes máquinas e que este efeito não inflacione a estimativa do erro experimental. Ou seja, calcula-se o efeito da diferença entre os dias da semana ou da diferença entre as máquinas e este efeito é reduzido do erro experimental. O fracionamento permite realizar apenas uma fração dos ensaios a serem realizados, sem perder informações relevantes. Um projeto fatorial completo permite estudar os efeitos principais e todas as interações entre os fatores controláveis. Com o aumento do número de fatores, o número de interações entre os fatores aumenta rapidamente. No entanto as interações de alta ordem são difíceis de interpretar e em geral não são significativas. Desta forma pode-se optar por rodar um projeto fatorial fracionado executando apenas uma fração dos ensaios e obtendo as mesmas informações relevantes do projeto fatorial completo, ou seja, os efeitos principais e os efeitos de interação de dois fatores As informações que são perdidas no projeto fatorial fracionado são as interações de mais alta ordem, mas que a princípio não são significativas (RIBEIRO; CATEN, 2003).

Montgomery (1997) afirma que antes de iniciar a experimentação é importante estabelecer o planejamento dos experimentos. Esse autor ressalta a importância do domínio do problema por parte de todas as pessoas envolvidas no experimento e, recomenda que durante a execução o processo seja cuidadosamente monitorado para garantir que tudo seja realizado de acordo com o planejado.

Na Figura 3 pode ser verificada a estratégia utilizada para a execução do experimento. Na etapa do objetivo, são definidas as variáveis de resposta a serem consideradas na otimização do experimento. Uma vez definida as variáveis de resposta, é realizado o planejamento do experimento que irá definir os ensaios a serem realizados.

\section{Figura 3 - Estratégia de um experimento}

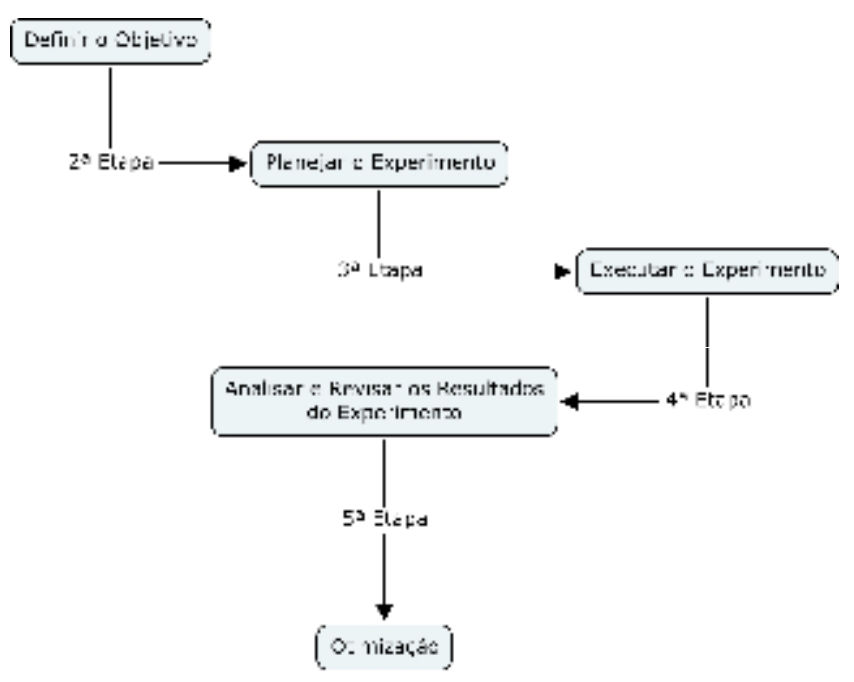


Os passos da etapa do planejamento de experimento são: (i) definição das variáveis de resposta que avaliam o desempenho de um experimento; (ii) definição dos parâmetros do processo que podem ter influência sobre as variáveis de resposta; (iii) priorização dos fatores controláveis que são um subconjunto dos parâmetros do processo que serão investigados no experimento; (iv) definição dos fatores constantes, subconjunto dos parâmetros do processo que não serão investigados no experimento; (v) definição do intervalo de investigação dos fatores controláveis); (vi) definição dos níveis dos fatores controláveis; (vii) definição das restrições experimentais; (viii) definição da necessidade de blocagem e/ou fracionamento; e (ix) definição da matriz experimental ser executada, ou seja, os ensaios com a combinação dos níveis dos fatores controláveis a serem realizados.

Conforme Figura 3, uma vez executado os ensaios, os dados coletados serão analisados utilizando-se ferramentas estatísticas para identificar quais os fatores controláveis possuem efeito significativo sobre cada uma das variáveis de resposta separadamente. Realizada a análise individual de cada variável de resposta, é realizada a otimização, ou seja, a definição dos ajustes dos níveis dos fatores controláveis que maximiza o desempenho do conjunto de variáveis de resposta simultaneamente.

O planejamento de experimentos possui como principais vantagens: (i) a diminuição do número de ensaios; (ii) o estudo de um número considerável de fatores; (iii) a detecção das interações entre fatores; (iv) a detecção dos níveis ótimos; (v) a melhoria de precisão dos resultados; e (vi) a otimização do experimento considerando as variáveis de resposta definidas inicialmente.

\section{Estudo aplicado}

\subsection{Determinação dos parâmetros do experimento}

O objetivo do experimento foi identificar os fatores controláveis que possuem efeito significativo sobre a variável de resposta tempo de cocção do produto.

Os fatores controláveis considerados que podem possuir algum efeito significativo sobre o tempo de cocção foram: (a) nível de carvão; (b) quantidade de espetos a ser assados; (c) tipo de carne; (d) altura dos espetos; e (e) ponto de cocção. Foram fixados dois níveis (baixo e alto) para cada fator controlável conforme Tabela 1.

Tabela 1 - Fatores controláveis e respectivos níveis

\begin{tabular}{|c|c|c|c|}
\hline Fatores & Fatores & \multicolumn{2}{c|}{ Níveis } \\
\hline A & Nível de Carvão & Baixo & Alto \\
\hline B & Número de Espetos & 4 & 2 \\
\hline
\end{tabular}




\begin{tabular}{|c|ccc|}
\hline C & Tipo de Carne & Carne Bovina & Carne de Frango \\
\hline D & Altura dos espetos & Baixo & Alto \\
\hline E & Estado da Carne & Mal Passado & Cozido \\
\hline $\boldsymbol{V R}$ & Tempo para assar & & \\
\hline
\end{tabular}

Os fatores que não foram controlados durante o experimento foram mantidos constantes para a redução do erro experimental. Foi utilizado o mesmo corte e marca de carne bovina e o mesmo produto para acender o carvão, neste caso, o álcool sólido marca $L U M I X$ e, a mesma marca de carvão RAIO SUL (carvão vegetal, lenha nativa mista procedente do estado do Paraná). Os experimentos foram realizados no mesmo ambiente para que o fator climático fosse mantido de forma inalterável. O auxiliar de pesquisa que fez o acendimento do carvão foi sempre o mesmo, evitando assim, a alteração dos procedimentos operacionais no experimento. A contagem de tempo foi iniciada no momento em que começou a aparecer as primeiras chamas.

Determinada a primeira parte de caracterização dos fatores controláveis do experimento, o seguinte passo foi a escolha do tipo de planejamento que se adapte melhor as quantidades de fatores e restrições experimentais. Quando existem muitos fatores, um experimento fatorial completo com todas as combinações possíveis dos níveis dos fatores envolve um grande número de ensaios, mesmo quando somente dois níveis de cada fator estão sendo pesquisados. Nesses casos, faz-se útil um plano que exija menos ensaios do que o experimento fatorial completo chamado de projeto fatorial fracionado. A fração é um subgrupo, cuidadosamente prescrito, de todas as combinações possíveis. A análise dos projetos fatoriais fracionários é relativamente direta, e a utilização de um fatorial fracionário não impede a possibilidade de uma complementação posterior de todo o experimento fatorial (SILVA, 2007).

A técnica de planejamento fatorial fracionado $2^{k-p}$ ( $k$ é o número de fatores de controle do experimento e $p$ é o número de colunas inseridas na matiz experimental) tem grande potencial de aplicação em problemas industriais, já que, com essa técnica, consegue-se, com uma pequena quantidade de ensaios, analisar a influência de um número grande de fatores.

Neste estudo utilizou-se um projeto fatorial fracionado $2^{5-1}$, com os fatores já mencionados. O projeto fatorial completo $2^{5}$ envolve 32 ensaios, os quais serão fracionados em dois, obtendo-se o projeto fatorial fracionado $2^{K-1}=2^{5-1}$ que contempla apenas 16 ensaios. Para a blocagem e posterior fracionamento foi utilizado o contraste da interação de mais alta ordem ABCDE.

A Tabela 2 apresenta a matriz experimental do projeto fatorial fracionado $2^{5-1}$ e os valores coletados para a variável de resposta tempo de cocção na última coluna. 
Tabela 2 - Matriz experimental do projeto

\begin{tabular}{|c|c|c|c|c|c|c|}
\hline Trat & $\mathbf{C}$ & $\boldsymbol{A C}$ & $\boldsymbol{D}$ & $\mathbf{C D}$ & $\boldsymbol{E}$ & $\boldsymbol{V} \boldsymbol{R}$ \\
\hline $\mathbf{1}$ & $(1)$ & 1 & $(1)$ & 1 & $(1)$ & $\mathbf{1 0}$ \\
\hline $\mathbf{a}$ & $(1)$ & $(1)$ & $(1)$ & 1 & $(1)$ & $\mathbf{5}$ \\
\hline $\mathbf{b}$ & $(1)$ & 1 & $(1)$ & 1 & $(1)$ & $\mathbf{1 0}$ \\
\hline $\mathbf{a b}$ & $(1)$ & $(1)$ & $(1)$ & 1 & $(1)$ & $\mathbf{5}$ \\
\hline $\mathbf{c}$ & 1 & $(1)$ & $(1)$ & $(1)$ & $(1)$ & $\mathbf{1 0}$ \\
\hline ac & 1 & 1 & $(1)$ & $(1)$ & $(1)$ & $\mathbf{1 8}$ \\
\hline bc & 1 & $(1)$ & $(1)$ & $(1)$ & $(1)$ & $\mathbf{1 1}$ \\
\hline abc & 1 & 1 & $(1)$ & $(1)$ & $(1)$ & $\mathbf{1 8}$ \\
\hline $\mathbf{d}$ & $(1)$ & 1 & 1 & $(1)$ & $(1)$ & $\mathbf{1 6}$ \\
\hline ad & $(1)$ & $(1)$ & 1 & $(1)$ & $(1)$ & $\mathbf{7}$ \\
\hline bd & $(1)$ & 1 & 1 & $(1)$ & $(1)$ & $\mathbf{1 5}$ \\
\hline abd & $(1)$ & $(1)$ & 1 & $(1)$ & $(1)$ & $\mathbf{7}$ \\
\hline cd & 1 & $(1)$ & 1 & 1 & $(1)$ & $\mathbf{1 6}$ \\
\hline bcd & 1 & $(1)$ & 1 & 1 & $(1)$ & $\mathbf{1 6}$ \\
\hline ae & $(1)$ & $(1)$ & $(1)$ & 1 & 1 & $\mathbf{9}$ \\
\hline abe & $(1)$ & $(1)$ & $(1)$ & 1 & 1 & $\mathbf{1 0}$ \\
\hline ce & 1 & $(1)$ & $(1)$ & $(1)$ & 1 & $\mathbf{1 1}$ \\
\hline ace & 1 & 1 & $(1)$ & $(1)$ & 1 & $\mathbf{2 8}$ \\
\hline bce & 1 & $(1)$ & $(1)$ & $(1)$ & 1 & $\mathbf{1 1}$ \\
\hline abce & 1 & 1 & $(1)$ & $(1)$ & 1 & $\mathbf{2 8}$ \\
\hline de & $(1)$ & 1 & 1 & $(1)$ & 1 & $\mathbf{2 5}$ \\
\hline ade & $(1)$ & $(1)$ & 1 & $(1)$ & 1 & $\mathbf{1 0}$ \\
\hline bde & $(1)$ & 1 & 1 & $(1)$ & 1 & $\mathbf{2 5}$ \\
\hline abde & $(1)$ & $(1)$ & 1 & $(1)$ & 1 & $\mathbf{1 0}$ \\
\hline cde & 1 & $(1)$ & 1 & 1 & 1 & $\mathbf{2 5}$ \\
\hline acde & 1 & 1 & 1 & 1 & 1 & $\mathbf{3 5}$ \\
\hline bcde & 1 & $(1)$ & 1 & 1 & 1 & $\mathbf{2 5}$ \\
\hline
\end{tabular}

\subsection{Execução e análise do experimento}

Uma vez executados os ensaios e mensurada a variável de resposta tempo de cocção, realizou-se a análise do experimento.

Os resultados obtidos foram analisados utilizando-se a ferramenta de análise de regressão. Análise de regressão é uma metodologia estatística que utiliza as relações entre duas ou mais variáveis quantitativas (ou qualitativas) de tal forma que uma variável pode ser predita a partir da outra ou outras. A análise de regressão representa os dados através de um modelo linear aditivo, onde o modelo inclui um componente sistemático e um aleatório.

$$
Y=f(X)+\varepsilon
$$

$f$ descreve a relação entre $X$ e $Y$. $\varepsilon$ são os erros aleatórios;

$Y=$ variável resposta ou dependente;

$X=$ variável independente ou variável regressora. 
Os dados podem ser obtidos a partir de duas situações: (i) dados experimentais: as observações $X$ e $Y$ são planejadas como o resultado de um experimento; e (ii) dados observacionais: observa-se os valores de $X$ e $Y$, nenhuma delas sob controle.

Segundo Ribeiro e Caten (2003), quando existem dados coletados (pares de valores) associando uma variável de resposta $\mathrm{Y}$ (variável dependente) com uma variável regressora $\mathrm{X}$ (variável independente), a variável de resposta Y pode ser descrita pelo seguinte modelo:

$$
Y=\beta_{0}+\beta_{1} X+\varepsilon
$$

onde $\varepsilon$ representa o termo de erro aleatório, com média 0 e $\sigma^{2}$

$\mathrm{Na}$ análise de regressão foram estudos os efeitos principais dos fatores controláveis e as interações de dois fatores. A equação de regressão é representada na Tabela 3, na qual se pode observar que os efeitos significativos foram os efeitos principais C (tipo de carne) D (altura dos espetos) e E (estado da carne) e as interações de dois fatores AC (nível de carvão e tipo de carne) e CD (tipo de carne e altura dos espetos). A Tabela 3 apresenta os efeitos considerados significativos, ou seja, aqueles cujo valor-p foram menores do que $5 \%$.

Tabela 3 -Valor de P

\begin{tabular}{|l|r|rrr|}
\hline & Coeficientes & Erro padrão & \multicolumn{1}{c|}{ Stat t } & valor-p \\
\hline Interseção & 17,052 & 0,479 & 35,630 & 0,000000000000000 \\
\hline C & 4,345 & 0,470 & 9,246 & 0,000000007510525 \\
\hline AC & 5,302 & 0,479 & 11,078 & 0,000000000313325 \\
\hline D & 3,095 & 0,470 & 6,586 & 0,000001601563803 \\
\hline CD & 1,427 & 0,479 & 2,981 & 0,007122670936912 \\
\hline E & 3,313 & 0,464 & 7,146 & 0,000000479224123 \\
\hline
\end{tabular}

A equação que relaciona a variável de resposta tempo de cocção com os fatores controláveis significativos é:

$$
Y=17,052+4,345 \times C+5,302 \times A C+3,095 \times D+1,427 \times C D+3,313 \times E
$$

$\mathrm{Na}$ Tabela 4, são apresentados os resultados obtidos para o coeficiente de determinação $\mathrm{R}^{2} \mathrm{e}$ $\mathrm{R}^{2}$ ajustado.

Tabela 4 - Cálculo do fator de determinação $\mathrm{R}^{2}$

\begin{tabular}{|l|r|}
\hline \multicolumn{2}{|c|}{ Estatística de regressão } \\
\hline R múltiplo & 0,9651 \\
\hline R-Quadrado & 0,9314 \\
\hline R-quadrado ajustado & 0,9150 \\
\hline Erro padrão & 2,3683 \\
\hline Observações & 27 \\
\hline
\end{tabular}

O fator de determinação $\mathrm{R}^{2}$ é igual a 0,9314 , ou seja, 93,14\% da variabilidade nos resultados do tempo de cocção da carne é devida ao efeito principal do fator $\mathrm{C}$ (tipo de carne bovino ou frango), do fator D (altura dos espetos baixo e alto) e do fator E (estado da carne passado ou mal 
passado) e ao efeito de interações AC (nível do carvão e tipo de carne) e CD (tipo de carne e altura dos espetos). A variabilidade restante, $6,86 \%$ da variabilidade do tempos de cocção, é devido a outros fatores que não foram estudados neste experimento.

$\mathrm{Na}$ Tabela 5, apresentam-se os valores da variável de resposta tempo obtida experimentalmente e os valores previstos utilizando a equação de regressão. Pode-se observar que os dados são bastante ajustados, corroborando o alto valor do coeficiente de determinação $\mathrm{R}^{2}$.

Tabela 5 - Valores previstos usando a equação da regressão linear

\begin{tabular}{|c|c|c|c|c|c|c|c|}
\hline Trata. & $\mathbf{C}$ & $\mathbf{A C}$ & $\mathbf{D}$ & $\mathbf{C D}$ & $\mathbf{E}$ & $\mathbf{V R}$ & Equação \\
\hline $\mathbf{1}$ & $(1)$ & 1 & $(1)$ & 1 & $(1)$ & $\mathbf{1 0}$ & $\mathbf{1 3}$ \\
\hline $\mathbf{a}$ & $(1)$ & $(1)$ & $(1)$ & 1 & $(1)$ & $\mathbf{5}$ & $\mathbf{2}$ \\
\hline $\mathbf{b}$ & $(1)$ & 1 & $(1)$ & 1 & $(1)$ & $\mathbf{1 0}$ & $\mathbf{1 3}$ \\
\hline $\mathbf{a b}$ & $(1)$ & $(1)$ & $(1)$ & 1 & $(1)$ & $\mathbf{5}$ & $\mathbf{2}$ \\
\hline $\mathbf{c}$ & 1 & $(1)$ & $(1)$ & $(1)$ & $(1)$ & $\mathbf{1 0}$ & $\mathbf{8}$ \\
\hline $\mathbf{a c}$ & 1 & 1 & $(1)$ & $(1)$ & $(1)$ & $\mathbf{1 8}$ & $\mathbf{1 9}$ \\
\hline $\mathbf{b c}$ & 1 & $(1)$ & $(1)$ & $(1)$ & $(1)$ & $\mathbf{1 1}$ & $\mathbf{8}$ \\
\hline abc & 1 & 1 & $(1)$ & $(1)$ & $(1)$ & $\mathbf{1 8}$ & $\mathbf{1 9}$ \\
\hline $\mathbf{d}$ & $(1)$ & 1 & 1 & $(1)$ & $(1)$ & $\mathbf{1 6}$ & $\mathbf{1 6}$ \\
\hline ad & $(1)$ & $(1)$ & 1 & $(1)$ & $(1)$ & $\mathbf{7}$ & $\mathbf{6}$ \\
\hline bd & $(1)$ & 1 & 1 & $(1)$ & $(1)$ & $\mathbf{1 5}$ & $\mathbf{1 6}$ \\
\hline abd & $(1)$ & $(1)$ & 1 & $(1)$ & $(1)$ & $\mathbf{7}$ & $\mathbf{6}$ \\
\hline $\mathbf{c d}$ & 1 & $(1)$ & 1 & 1 & $(1)$ & $\mathbf{1 6}$ & $\mathbf{1 7}$ \\
\hline bcd & 1 & $(1)$ & 1 & 1 & $(1)$ & $\mathbf{1 6}$ & $\mathbf{1 7}$ \\
\hline ae & $(1)$ & $(1)$ & $(1)$ & 1 & 1 & $\mathbf{9}$ & $\mathbf{9}$ \\
\hline abe & $(1)$ & $(1)$ & $(1)$ & 1 & 1 & $\mathbf{1 0}$ & $\mathbf{9}$ \\
\hline ce & 1 & $(1)$ & $(1)$ & $(1)$ & 1 & $\mathbf{1 1}$ & $\mathbf{1 5}$ \\
\hline ace & 1 & 1 & $(1)$ & $(1)$ & 1 & $\mathbf{2 8}$ & $\mathbf{2 5}$ \\
\hline bce & 1 & $(1)$ & $(1)$ & $(1)$ & 1 & $\mathbf{1 1}$ & $\mathbf{1 5}$ \\
\hline abce & 1 & 1 & $(1)$ & $(1)$ & 1 & $\mathbf{2 8}$ & $\mathbf{2 5}$ \\
\hline de & $(1)$ & 1 & 1 & $(1)$ & 1 & $\mathbf{2 5}$ & $\mathbf{2 3}$ \\
\hline ade & $(1)$ & $(1)$ & 1 & $(1)$ & 1 & $\mathbf{1 0}$ & $\mathbf{1 2}$ \\
\hline bde & $(1)$ & 1 & 1 & $(1)$ & 1 & $\mathbf{2 5}$ & $\mathbf{2 3}$ \\
\hline abde & $(1)$ & $(1)$ & 1 & $(1)$ & 1 & $\mathbf{1 0}$ & $\mathbf{1 2}$ \\
\hline $\mathbf{c d e}$ & 1 & $(1)$ & 1 & 1 & 1 & $\mathbf{2 5}$ & $\mathbf{2 4}$ \\
\hline acde & 1 & 1 & 1 & 1 & 1 & $\mathbf{3 5}$ & $\mathbf{3 5}$ \\
\hline bcde & 1 & $(1)$ & 1 & 1 & 1 & $\mathbf{2 5}$ & $\mathbf{2 4}$ \\
\hline & & & & & & & \\
\hline
\end{tabular}

$\mathrm{Na}$ seqüência, apresenta-se o gráfico dos fatores que resultaram significativos na análise realizada. Na Figura 4, pode-se observar o efeito do fator tipo de carne sobre a variável de resposta tempo de cocção, onde se pode concluir que a carne de frango demora mais tempo para cozer. 
Figura 4 - Plotagem do Fator C em função da V.R.

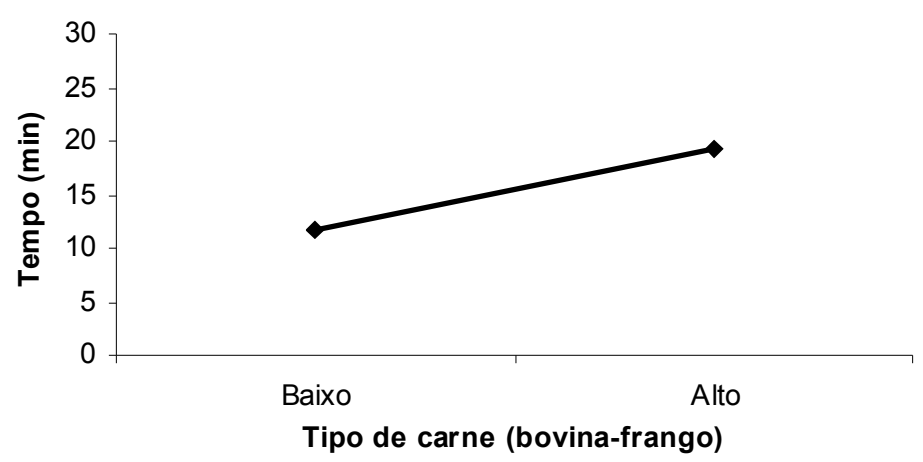

A Figura 5 apresenta o efeito de interação AC, onde se pode verificar que para o nível baixo de carvão não existe diferença de tempo de cozimento entre os dois tipos de carne. No entanto, no nível alto de carvão, a carne de frango cozinha em menor tempo do que a carne bovina.

Figura 5 - Plotagem do Fator AC em função da V.R.

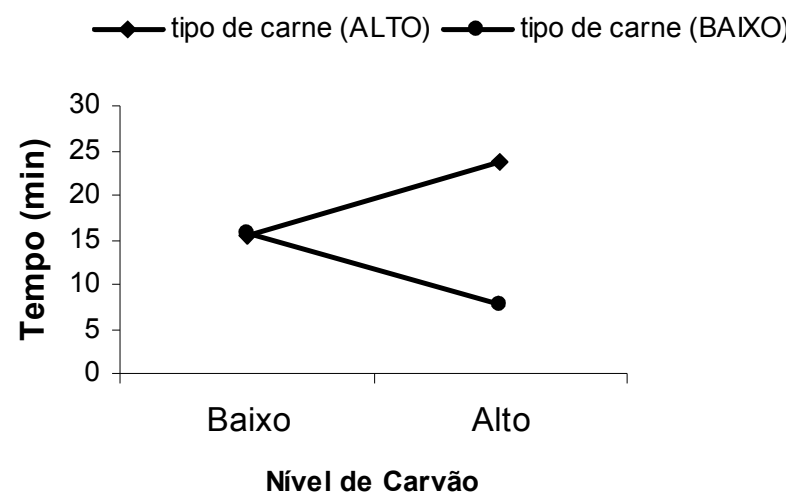

Na Figura 6, o tempo de cocção aumenta dependendo da altura na qual se encontram assando os espetos, a maior altura é o maior tempo que a carne demora a assar.

Figura 6 - Plotagem do Fator D em função da V.R.

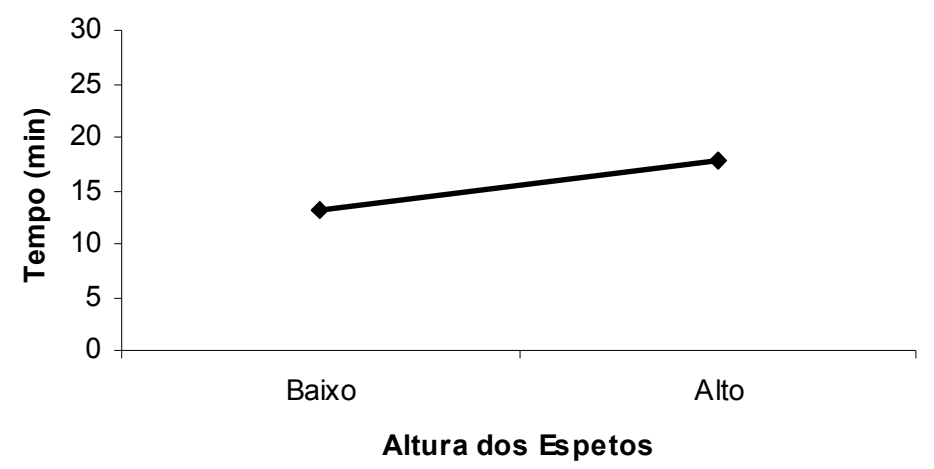


A Figura 7 apresenta o efeito de interação $C D$, onde se pode verificar que a diferença no tempo de cozimento entre o tipo de carne é amplificada quando os espetos estão no nível alto.

Figura 7 - Plotagem do Fator CD em função da V.R.

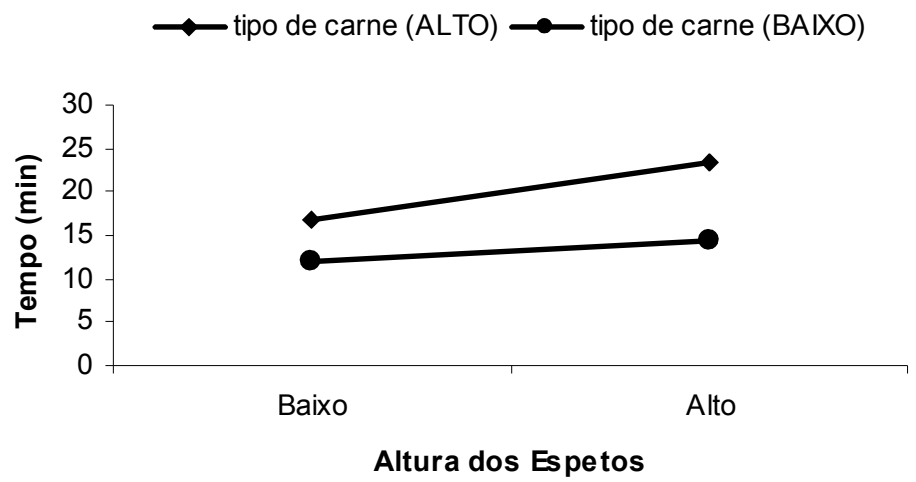
A Figura
8 apresenta 0

efeito principal do fator E, estado da carne. Pode-se verificar que quanto maior a altura dos espetos maior o tempo de cocção.

Figura 8 - Plotagem do Fator E em função da V.R.

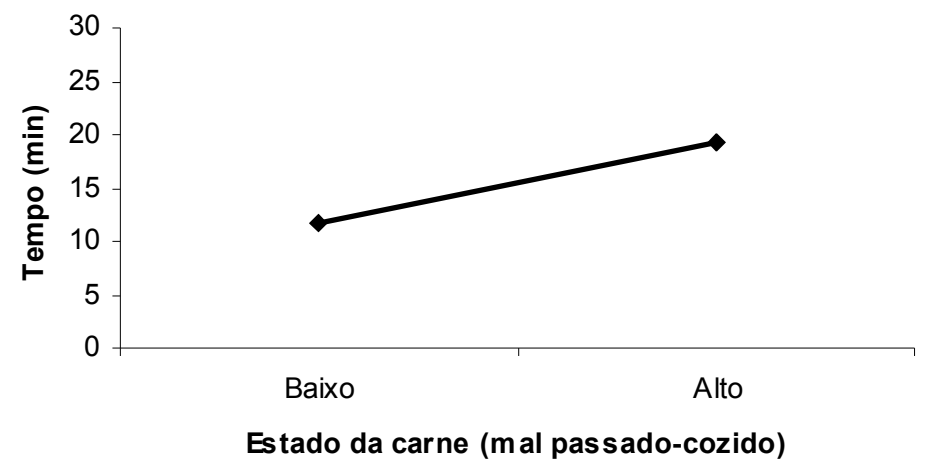

\subsection{Análise de validade do modelo}

Segundo Ribeiro e Caten (2003), a adequação do ajuste e as suposições do modelo podem ser verificadas através de uma análise dos resíduos. Os resíduos padronizados são calculados como sendo a diferença entre os valores observados e valores previstos pela equação dividindo-se pelo desvio-padrão, como pode ser visto na equação a seguir:

$$
R_{i}=\frac{Y_{i}-\left(b_{0}+b_{1} X_{i}\right)}{S}
$$

onde :

$$
S=\sqrt{\frac{S Q R}{n-2}} \quad \text { e } \quad S Q R=S Y Y-b_{1} S X Y
$$


sendo:

$R_{i}=$ residuos padronizados;

$S Y Y=$ Soma quadrada do fator da variável de resposta $Y$;

$S X Y=$ Soma quadrada das interações entre a variável dependente e independente;

$S Q R=$ Soma quadrada dos resíduos.

A adequação do ajuste é testada plotando-se os resíduos padronizados $R_{i}$ em função de $\mathrm{X}$ como pode ser visto nas Figuras 9 a 11. Foram considerados dados atípicos aqueles que apresentam os resíduos padronizados $R_{i}$ maiores do que $\mathrm{Z}= \pm 1,96$, correspondente a um nível de significância de $5 \%$.

Figura 9 - Plotagem de resíduos padronizados Fator C

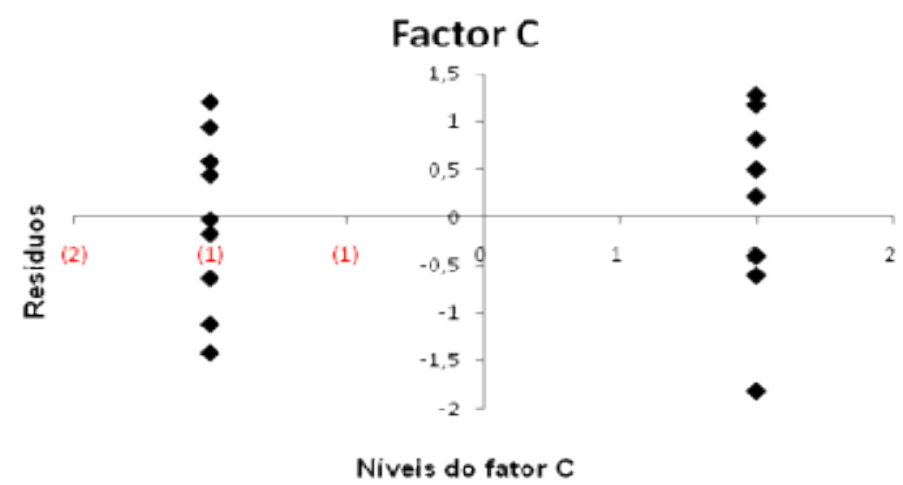

Figura 10 - Plotagem de resíduos padronizados Fator D

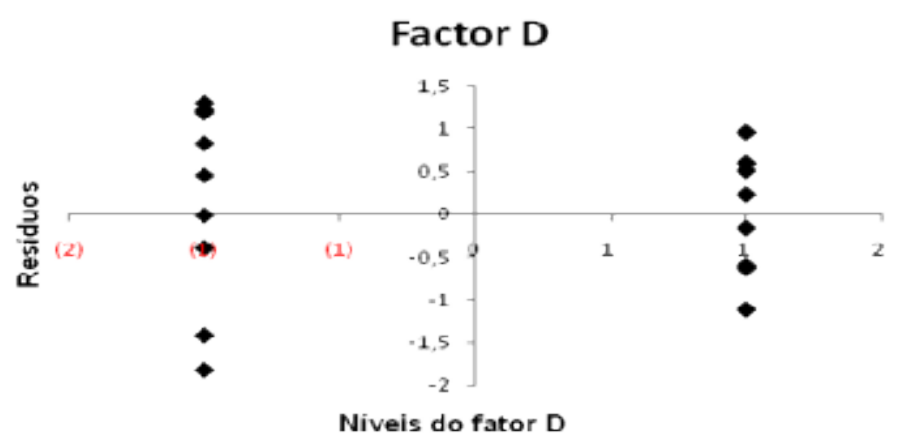




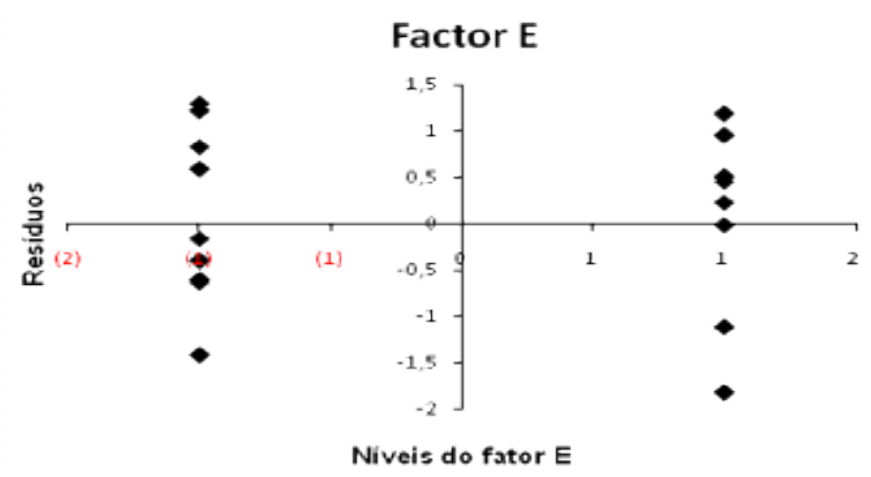

Como se pode observar nos gráficos anteriores, os resíduos encontram-se distribuídos aleatoriamente em torno do 0 , o que representa um ajuste adequado do modelo. Não foram encontrados dados atípicos.

\section{Conclusões}

Este artigo apresentou os resultados da aplicação da técnica de planejamento e análise de experimentos a uma inovação tecnológica, churrasqueira portátil, construída a partir da reutilização de um botijão de gás para refrigeração, que é considerado um resíduo após a utilização do gás.

O objetivo do experimento foi determinar quais os fatores que influenciam na variável de resposta tempo de cocção para assar distintos tipos de carne na churrasqueira portátil. Os fatores controláveis investigados neste estudo foram: (a) nível de carvão; (b) quantidade de espetos a serem assados; (c) tipo de carne; (d) altura dos espetos; e (e) ponto de cocção. A variável de resposta de saída é representada pelo tempo de cocção do produto. Para cada fator controlável foram investigados dois níveis (baixo e alto).

O planejamento do experimento foi realizado utilizando-se a ferramenta de Projeto de Experimentos. Foi utilizado um projeto fatorial fracionado $2^{5-1}$ sem repetições contemplando 16 ensaios. Os resultados foram analisados utilizando-se a análise de regressão onde foram investigadas, além dos fatores principais, o efeito das interações de dois fatores.

A análise dos resultados foi realizada utilizando-se a ferramenta de Análise de Regressão. Os fatores considerados significativos foram os efeitos principais do fator C (tipo de carne), D (altura dos espetos) e E (estado da carne) e os efeitos das interações de dois fatores AC (nível de carvão e tipo de carne) e CD (tipo de carne e altura dos espetos).

Com a equação de regressão é possível prever os tempos de cocção para cada uma das combinações dos níveis dos fatores controláveis. 


\begin{abstract}
This paper describes the results obtained by applying Design of Experiments (DOE) systematic to a technological innovation - a portable barbeque - constructed from a recycled refrigeration gas cylinder normally considered waste when empty. In the planning of the experiment we used a project involving factorial fractions $2^{5-1}$ without repetitions but incorporating a total of 16 tests. .The results were analyzed by regression analyses and, in addition to the principal factors, the interaction of the two factors was investigated. With the regression equation it is possible to predict the cooking times for each one of the combinations of the levels of the controllable factors.
\end{abstract}

Key-words: design of experiments, innovation, linear regression, project $2^{\mathrm{k}-\mathrm{p}}$, optimization.

\title{
Referências
}

BERTONCELLO, I; GOMES, L. V. N. Análise diacrônica e sincrônica da cadeira de rodas mecanomanual. Produção. 2002, vol.12, no.1, p.72-82.

BONSIEPE, G. Teoria y práctica del diseño industrial. Barcelona: Gustavo Gili, 1978.

BONDUELLE, A. Caractérisation du panneau de particules surface mélaminé par son aptitude a I'usinabilité. Doctorate Tesis 205 p., Université de Nncy I. Nancy, 1994.

COLENCI, A. T. O ensino de engenharia como uma atividade de serviços: a exigência de atuação em novos patamares de qualidade acadêmica. Dissertação (Mestrado em Engenharia de Produção). Escola de Engenharia de São Carlos. USP- Universidade de São Paulo, 2000.

DANILEVICZ, A. M. F. Modelo para condução de decisões estratégicas associadas ao gerenciamento da inovação em produtos. 231 p. Tese (Doutorado em Engenharia de Produção). Escola de Engenharia. Programa de PósGraduação em Engenharia de Produção. Universidade Federal do Rio Grande do Sul, Porto Alegre, 2006.

ELSAYED, E. A.; CHEN, A. Optimal levels of process parameters for products with multiples characteristics. International Journal Production Research. v. 31, n. 5, 1993.

GALDÁMEZ, E. V. C. Aplicação das téenicas de planejamento e análise de experimentos na melhoria da qualidade de um processo de fabricação de produtos plásticos. Dissertação (Mestrado em Engenharia de Produção). Escola de Engenharia de São Carlos. USP - Universidade de São Paulo, 2002.

HENNIG, G. J. Metodologia do ensino de ciências. 2. ed. Porto Alegre: Mercado Aberto, 1994.

HERNÁNDEZ, F.; SANCHO, J. M.; CARBONELL, J.; TORT, A.; SIMÓ, N.; SÁNCHEZ-CORTÉS, E. Aprendendo com as inovações nas escolas. Trad. Ernani Rosa. Porto Alegre: Artes Médicas Sul, 2000.

HORD. Evaluating educational innovation. New York: Croom Helm, 1987.

KUCZMARSKI, T. D. Por uma consciência inovadora. HSM Management. n. 5. jan/fev, p. 62-68, 1998.

MONTGOMERY, D. C. Design and analysis of experiments. 5. ed. New York: John Wiley \& Sons, 1997.

SILVA, M. B. Projeto de experimentos. Disponível em: <http://www.eel.usp.br /copg/des_arq/messias/IntroduoProjeto\%20Experimentos.pdf >. Acesso em: 22 de Junho de 2007.

PHADKED, M. S. Quality engineering using robust design. New Jersey: Prentice Hall, 1989.

PIZZOLATO, M.; CATEN, C. S. ten; FOGLIATTO, F. S. Definition of the warranty period of an experimentally optimized product. Gestão \& Produção, São Carlos, v. 12, n. 2, 2005. 
RIBEIRO, J. L. D.; CATEN, C. S. ten. Projeto de experimentos. Porto Alegre: Universidade Federal do Rio Grande do Sul, Escola de Engenharia. Programa de Pós Graduação em Engenharia de Produção, 2003.

ROZENFELD, H.; FORCELlini, F. A.; AMARAL, D. C.; TOLEDO, J. C.; SILVA, S. L.; ALLIPRANDINI, D. H.; SCALICE, R. K. Gestão de desenvolvimento de produtos: uma referência para a melhoria do processo. São Paulo: Saraiva, 2006.

\section{Dados dos autores:}

Nome completo:Mariela Haidée Aranda

Filiação institucional:Universidade Federal do Rio Grande do Sul

Departamento:Engenharia de Produção

Função ou cargo ocupado:Mestranda

Endereço: Av. Borges de Medeiros 658, ap: 1202, Bairro: Centro;

CEP: 90020-022, Cidade: Porto Alegre; Estado: Rio Grande do Sul; País: Brasil;

Telefones para contato:(51) 30128433

e-mail:mariela@producao.ufrgs.br

Nome completo: Carlos Fernando Jung

Filiação institucional: Universidade Federal do Rio Grande do Sul - UFRGS

Departamento: Programa de Pós-Graduação em Engenharia de Produção - PPGEP

Função ou cargo ocupado: Doutorando em Engenharia de Produção

Endereço completo para correspondência (bairro, cidade, estado, país e CEP):

Avenida Osvaldo Aranha, 99, $5^{\circ}$ Andar, Porto Alegre, RS, Brasil, 90035-190

Telefones para contato: 51.99723926

e-mail: carlosfernandojung@gmail.com

Nome completo: Carla Schwengber ten Caten

Filiação institucional: Universidade Federal do Rio Grande do Sul - UFRGS

Departamento: Programa de Pós-Graduação em Engenharia de Produção - PPGEP

Função ou cargo ocupado: Professora Adjunta

Endereço completo para correspondência (bairro, cidade, estado, país e CEP):

Avenida Osvaldo Aranha, 99, $5^{\circ}$ Andar, Porto Alegre, RS, Brasil, 90035-190

Telefones para contato: 51.33083491

e-mail: tencaten@producao.ufrgs.br

Recebido para publicação em: 18/07/2008

Aceito para publicação em: 09/09/2008 